\author{
MITSUBISHI ELECTRIC RESEARCH LABORATORIES \\ http://www.merl.com
}

\title{
Antenna Subset Selection in Measured Indoor Channels
}

\author{
P. Almers, T. Santos, F. Tufvesson, A.F. Molisch, J. Karedal and A.J. Johansson
}

TR2007-103 October 2008

\begin{abstract}
Antenna subset selection can greatly reduce the implementation complexity of multiple input multiple output (MIMO) systems while retaining most of their benefits. This paper investigates the diversity gain and capacity of such systems in wireless personal area networks. Considered scenarios include both the communication between access point to a laptop, and between two hand held devices. We analyze the performance of different antenna selection algorithms and signal combining methods in measured dual-polarized narrow band and wide band propagation channels. We find that line-of-sight and non-line-of-sight situations have fairly similar behavior. Different polarizations result in similar signal-to-noise ratio gains when the multiple antennas are used for diversity, but result in noticeably different capacities in spatial-multiplexing systems. We also find that radio frequency (RF) preprocessing of the signals is less effective for hand held handsets with non-uniform antenna arrangements than for uniform linear arrays. For communications between hand held devices, simple selection (of one out of four antennas) shows extremely high performance gains compared to no-selection. Finally, we compare bulk selection (same antenna subset is used for all frequency sub-channels) to per-tone selection (different antenna subsets can be used for each frequency sub-channel) for wide band channels. Bulk selection together with RF preprocessing performs almost a s well as per-tone selection for some scenarios.
\end{abstract}

IET Microwaves, Antennas \& Propagation

This work may not be copied or reproduced in whole or in part for any commercial purpose. Permission to copy in whole or in part without payment of fee is granted for nonprofit educational and research purposes provided that all such whole or partial copies include the following: a notice that such copying is by permission of Mitsubishi Electric Research Laboratories, Inc.; an acknowledgment of the authors and individual contributions to the work; and all applicable portions of the copyright notice. Copying, reproduction, or republishing for any other purpose shall require a license with payment of fee to Mitsubishi Electric Research Laboratories, Inc. All rights reserved.

Copyright (C) Mitsubishi Electric Research Laboratories, Inc., 2008

201 Broadway, Cambridge, Massachusetts 02139 



\title{
Antenna subset selection in measured indoor channels
}

\author{
P. Almers, T. Santos, F. Tufvesson, A.F. Molisch, J. Karedal and A.J. Johansson
}

\begin{abstract}
Antenna subset selection can greatly reduce the implementation complexity of multiple input multiple output (MIMO) systems while retaining most of their benefits. This paper investigates the diversity gain and capacity of such systems in wireless personal area networks. Considered scenarios include both the communication between access point to a laptop, and between two handheld devices. We analyse the performance of different antenna selection algorithms and signal combining methods in measured dual-polarised narrowband and wideband propagation channels. We find that line-of-sight and non-line-of-sight situations have fairly similar behaviour. Different polarisations result in similar signal-to-noise ratio gains when the multiple antennas are used for diversity, but result in noticeably different capacities in spatial-multiplexing systems. We also find that radiofrequency (RF) preprocessing of the signals is less effective for handheld handsets with non-uniform antenna arrangements than for uniform linear arrays. For communications between handheld devices, simple selection (of one out of four antennas) shows extremely high performance gains compared to no-selection. Finally, we compare bulk selection (same antenna subset is used for all frequency sub-channels) to per-tone selection (different antenna subsets can be used for each frequency sub-channel) for wideband channels. Bulk selection together with RF preprocessing performs almost as well as per-tone selection for some scenarios.
\end{abstract}

\section{Introduction}

During the last decade, wireless systems with multiple antennas at both receiver (RX) and transmitter (TX) side have attracted huge attention since they show great performance enhancement $[1,2]$. These so called multiple-input multiple-output (MIMO) systems exploit the multiple antennas to improve the data rate and/or the signal-to-noise ratio (SNR) of the system. However, the increased hardware complexity is a considerable disadvantage for deploying MIMO. Conventional MIMO systems require one downconversion/upconversion radio frequency (RF) chain for each antenna element. While the antenna elements themselves do not increase transceiver costs significantly, the RF chains are a very significant cost factor.

The increase in complexity has motivated antenna subset selection schemes, often called hybrid selection (HS) [3]. In HS schemes, only the signals belonging to a selected subset of antenna elements are chosen for upconversion/downconversion and processing, hence the number of RF chains can be reduced. The performance loss (compared to a fullcomplexity system with the same number of antenna elements) is relatively small; it has been shown that antenna selection retains the diversity order, while slightly reducing the beamforming gain.

Because of their attractive properties, MIMO systems with antenna selection have been investigated extensively in the academic literature. Reference [3] investigated

(C) The Institution of Engineering and Technology 2007

doi:10.1049/iet-map:20060306

Paper first received 8th November 2006 and in revised form 16th March 2007

P. Almers, T. Santos, F. Tufvesson, A.F. Molisch, J. Karedal and A.J. Johansson are with the Department of Electrical \& Information Technology, Lund University, Box 118, Lund SE-221 00, Sweden

A.F. Molisch is with Mitsubishi Electric Research Labs, 201 Broadway, Cambridge, MA 02139, USA, and also with the Department of Electrical \& Information Technology, Lund University, Box 118, Lund SE-221 00, Sweden E-mail: peter.almers@eit.lth.se
SNR, bit error rate (BER) and symbol error rate for a singleinput multiple-output (SIMO) system with a single element at the transmitter, and antenna subset selection together with maximum ratio combining (MRC) of the selected antenna elements at the receiver. Transmit diversity systems with $\mathrm{HS}$ at the transmitter and MRC at the receiver were analysed in [4]. Capacity bounds for the MIMO systems with HS at the receiver were analysed in [5], whereas HS for linear receivers was investigated by [6]. Subset selection combined with space-time coding was analysed in [7]. Finding the optimum subset requires an exhaustive search of all possible antenna combinations, hence fast selection algorithms are given in $[8,9]$. References $[10,11]$ suggested preprocessing of the signal in the RF domain (between antenna elements and selection switch) that can enhance the beamforming gain especially in correlated channels [12]. HS has also been included in the recent IEEE $802.11 \mathrm{n}$ standard draft for high-throughput wireless computer networks [13] and antenna selection performance has been evaluated in that context [14]. Further references and an overview of MIMO systems with HS and preprocessing can be found in [15-17].

Despite the large number of papers on the topic, only few results exist in the literature on HS/preprocessing evaluations based on measurements. Narrowband beam diversity selecting one beam (synthetic Butler matrix) was investigated in [18] and two beams in [19]. The work of Grau et al. [20] and Onizawa et al. [21], which were done independent of our investigation [22] showed analysis as well as measurement results of selection combing (one antenna selected) in combination with an implementation of a Butler matrix and Eigenbeam forming, respectively. Theoretical analyses mentioned above have been based on simplified channel models, including independent identically distributed (i.i.d.) complex Gaussian channels, and the 802.11n Kronecker channel models [23]; furthermore, they assume uniform linear arrays for the antenna configurations. Most papers (with the exception of the synthetic 
channels in [12]) also assume the channels to be frequency-flat.

These investigations give valuable insights into the fundamental behaviour of antenna selection. However, they do not allow to assess the performance in realistic situations where irregular antenna structures, dense multipath environments and shadowing by users play an important role.

The current paper tries to alleviate that problem, and analyses the performance of different antenna selection and preprocessing methods in measured propagation channels (Our recent paper [24] described the propagation characteristics of these channels; however, no analyses of antenna selection and diversity schemes were made in that contribution.). The analysed scenario is personal area networks (PANs) in an indoor office environment, where transmitter and receiver are separated by less than $10 \mathrm{~m}$. Communications between access point-to-PC/laptop (AP-PC) at $2.6 \mathrm{GHz}$, as well as handheld-to-handheld ( $\mathrm{HH}-\mathrm{HH})$ communication at $5.2 \mathrm{GHz}$, are investigated for both line-of-sight (LOS) and non-LOS (NLOS) (Fig. 1). The contributions of the paper are the following:

- a comprehensive investigation of the performance of seven antenna subset selection schemes, with and without preprocessing both in a diversity and capacity sense. Such a comparison has previously only been partially available in the literature even for simplified synthetic channel models.

- performance comparison in measured wireless personal area network (WPAN) channels covering both LOS and NLOS channels, and a comparison of the results with those of synthetic MIMO channel models.

- an analysis of the impact of polarisation and a comparison of spatial- with polarisation- diversity.

- the influence of antenna selection on diversity in wideband channels and the performance of different wideband selection schemes.

- the effect of the array element configuration, in particular, a comparison of the performance of uniform linear arrays (ULA) against that of irregular arrays, which is especially relevant for comparison.

The rest of the paper is organised the following way: sections 2 and 3 describe the system model and the selection algorithms, respectively. Section 4 describes the measurement setup and section 5 then compares the performance (both in terms of SNR and capacity) of seven different antenna selection/preprocessing schemes for line-of-sight (LOS) and non-line-of-sight (NLOS) scenarios in flatfading channels. In the subsequent section, we investigate the impact of frequency selective channels and we compare the effect of per-tone selection and bulk selection.

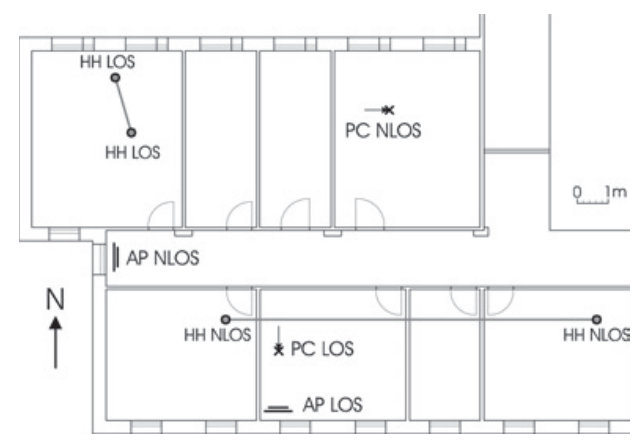

Fig. 1 Site map for the static AP-PC and HH-HH scenarios
Section 7, finally, investigates the impact of polarisation and antenna configuration effects on SNR gain and capacity. It also analyses the impact of shadowing on the different antenna arrangements. A summary and conclusion wrap up the paper.

\section{System model}

For the analysis, we consider a multiple antenna system with $N_{\mathrm{R}}$ receive and $N_{\mathrm{T}}$ transmit antenna elements from which a subset of $L_{\mathrm{R}}$ receive elements and $L_{\mathrm{T}}$ transmit elements is chosen; we use the short notation: $N_{\mathrm{R}}$ : $L_{\mathrm{R}} \times N_{\mathrm{T}}: L_{\mathrm{T}}$. For the analysis we assume a quasi-static channel; with the exception of section 6 , we also assume frequency-flat fading. The channel between the selected antenna elements is then described by the transfer matrix $\tilde{\boldsymbol{H}} \in \mathbb{C}^{L_{\mathrm{R}} \times L_{\mathrm{T}}}$, which is a $L_{\mathrm{R}} \times L_{\mathrm{T}}$ sized sub-matrix of the full channel matrix $\boldsymbol{H} \in \mathbb{C}^{N_{\mathrm{R}} \times N_{\mathrm{T}}}$. For later use, we denote the singular value decomposition of the channel transfer matrix $\tilde{\boldsymbol{H}}=\boldsymbol{U} \Sigma \boldsymbol{V}^{\dagger}$, where $\boldsymbol{U}$ and $\boldsymbol{V}^{\dagger}$ are unitary matrices representing the left and right singular vector spaces, and $\boldsymbol{\Sigma}$ is a diagonal matrix containing all the singular values. Further, $[\cdot]^{\dagger}$ denotes the Hermitian transpose, and $\lambda_{\tilde{\boldsymbol{H}}, i}$ denotes the $i$-largest singular value of $\boldsymbol{H}$. We analyse antenna selection systems that are designed either for maximising diversity, or for achieving maximum capacity. In the following, we establish the mathematical description of those systems.

For the diversity case, we assume full channel state information at the transmitter as might occur, for example, in a time-division duplex system, or a frequency-division duplex with explicit feedback [25]. The optimum system employs maximum-ratio combining (MRC) and maximumratio transmission (MRT) for the selected antenna elements at receiver and transmitter, respectively. Thus, the receivetransmit relation of the diversity scheme can be modelled as

$$
\boldsymbol{r}=\tilde{\boldsymbol{H}} v s+n
$$

where $\boldsymbol{r} \in \mathbb{C}^{L_{\mathrm{R}} \times 1}$ is the received vector; $\boldsymbol{v} \in \mathbb{C}^{L_{\mathrm{T}} \times 1}$, the transmit weighting vector and $\boldsymbol{n} \in \mathbb{C}^{L_{R} \times 1}$, the noise vector assumed to have an i.i.d. complex Gaussian distribution. Note that in this system model, the transmit data, $s$, is a scalar, that is, we transmit only a single data stream. This stream might have been encoded with a forward errorcorrecting code, but there is no space-time coding. At the RX side, the signal from the selected antenna elements is downconverted, weighted and combined with the receive weighting vector $\boldsymbol{u}^{\dagger}$. The optimal TX and RX weighting vectors $\boldsymbol{v}$ and $\boldsymbol{u}^{\dagger}$ are the conjugates of the singular vectors belonging to $\lambda_{\tilde{\boldsymbol{H}}, 1}$, that is, $\boldsymbol{v}_{\tilde{\boldsymbol{H}}, 1}$ and $\boldsymbol{u}_{\tilde{\boldsymbol{H}}, 1}^{\dagger}$ [26]. The SNR that can be achieved is $[26,27]$

$$
\gamma=\frac{\left|\boldsymbol{u}_{\tilde{\boldsymbol{H}}, 1}^{\dagger} \tilde{\boldsymbol{H}} \boldsymbol{v}_{\tilde{\boldsymbol{H}}, 1}\right|^{2}}{\left\|\boldsymbol{u}_{\tilde{\boldsymbol{H}}, 1}^{\dagger}\right\|^{2}}=\lambda_{\tilde{\boldsymbol{H}}, 1}^{2}
$$

The performance enhancement of the system utilising diversity can be divided into two categories: (i) increased average link gain, also called beamforming gain, resulting in a mean SNR gain of $N_{\mathrm{R}} \cdot N_{\mathrm{T}}$ in a pure LOS scenario, and (ii) diversity gain owing to a change of the slope of the bit error rate (BER) against SNR curve; this diversity gain depends on the considered level of the BER (or outage). For an NLOS situation, the diversity order is equal to the number of independent TX/RX elements, $N_{\mathrm{R}} \cdot N_{\mathrm{T}}$. We define the total diversity gain (at a specified outage level) as the decrease in SNR that is required for a multi-antenna 
system to achieve the same BER as a single-antenna system [25].

For a system that maximises the capacity, for example, by means of spatial multiplexing, the receive-transmit relation of the diversity scheme can be modelled as [2]

$$
\boldsymbol{r}=\tilde{\boldsymbol{H}} \boldsymbol{s}+\boldsymbol{n}
$$

Note that in this system model, the transmit data $s$ is a vector, $\boldsymbol{s} \in \mathbb{C}^{L_{\mathrm{T}} \times 1}$, that is, we transmit multiple data streams. At the RX side, the signals from the selected antenna elements are downconverted and processed, weighted and combined with the receive weighting matrix, $\boldsymbol{U}^{\dagger}$. The channel capacity is then found from [2]

$$
C=\sum_{\forall \lambda_{\tilde{\boldsymbol{H}}, k} \neq 0} \log _{2}\left(1+\gamma_{\mathrm{eval}} \frac{\lambda_{\tilde{\boldsymbol{H}}, k}^{2}}{L_{\mathrm{T}}}\right)
$$

\section{Antenna selections schemes}

As mentioned in the introduction, there are several selection schemes and preprocessing methods proposed in the literature. Furthermore, the optimum antenna elements can be selected in different ways. In the following, we list the algorithms that we compare in this paper.

\subsection{Full-compexity (FC)}

All available antenna elements are used. For a diversity system, the FC scheme results in full diversity order (e.g. $N_{\mathrm{R}} \cdot N_{\mathrm{T}}$ in the i.i.d. case) in addition to beamforming gain. In terms of capacity, the FC scheme results in full spatial multiplexing gain (under the assumption that $\boldsymbol{H}$ has full rank).

\subsection{Hybrid selection (HS)}

(1) Optimum Hybrid Selection (HS-B): A subset of antenna elements are selected and used for further processing. The only way to select the optimal antenna subset is an exhaustive search of all possible subsets and pick the one giving the best SNR [3] for the diversity case and the one giving the best capacity for the capacity evaluations [5]. Hence, HS-B at both link ends require $\left(\begin{array}{c}N_{\mathrm{R}} \\ L_{\mathrm{R}}\end{array}\right) \cdot\left(\begin{array}{l}N_{\mathrm{T}} \\ L_{\mathrm{T}}\end{array}\right)$ computations for each channel realisation.

(2) Power-based selection (PBS): In this case, the antennas are selected in a sub-optimum low complexity approach. The $L_{\mathrm{R}}$ strongest elements are selected from the average power received from all TX elements. Selection at the other link end is done analogous. The subset search involves $N_{\mathrm{R}}+N_{\mathrm{T}}$ computations compared to $\left(\begin{array}{c}N_{\mathrm{R}} \\ L_{\mathrm{R}}\end{array}\right) \cdot\left(\begin{array}{c}N_{\mathrm{T}} \\ L_{\mathrm{T}}\end{array}\right)$ for HS-B.

(3) Random and worst selection (HS-R and HS-W): For comparison, random selection (HS-R) and worst selection (HS-W) are also considered. In HS-R the subset is chosen randomly and in HS-W the worst of all possible subsets is selected as a worst case scenario.

\subsection{Phase shift preprocessing and selection (PSS)}

In order to improve the beamforming gain, a preprocessing of the received signals can be performed in the RF domain, that is, between the antenna elements and the selection switch [11]. The PSS preprocessing approach uses only variable phase shifters operating in the RF domain whose values are adjusted depending on the channel state information, but no variable-gain amplifiers. When applied to a diversity system, such a scheme, with two or more demodulators (PSS-opt), shows identical performance compared with the FC scheme. The normalised SNR becomes $\gamma_{\mathrm{PSS}-\mathrm{opt}}=\gamma_{\mathrm{FC}}$. For one demodulator (PSS-sopt), the normalised SNR that can be achieved is

$$
\gamma_{\text {PSS-sopt }}=\frac{\left|\boldsymbol{\phi}_{\boldsymbol{u}_{\boldsymbol{H}, 1}^{\dagger}}^{\dagger} \boldsymbol{H} \boldsymbol{\phi}_{\boldsymbol{v}_{\boldsymbol{H}, 1}}\right|^{2}}{N_{\mathrm{T}}}
$$

where the applied phase shift $\phi_{\boldsymbol{u}_{H, 1}}$ and $\phi_{\boldsymbol{v}_{H, 1}}$ are simple suboptimal approximations to the optimal vector $\boldsymbol{u}_{\boldsymbol{H}, 1}$ and $\boldsymbol{v}_{\boldsymbol{H}, 1}$, including only the phase information of the entries in $\boldsymbol{u}_{\boldsymbol{H}, 1}$ and $\boldsymbol{v}_{\boldsymbol{H}, 1}$. PSS-sopt is equivalent to equal gain combining (EGC) [28].

The capacity is evaluated in a similar way. However, the number of spatial streams after selection is smaller compared with $\mathrm{FC}$, hence $C_{\mathrm{PSS}-\mathrm{opt}} \leq C_{\mathrm{FC}}$. PSS-opt uses the min $\left(L_{\mathrm{R}}, L_{\mathrm{T}}\right)$ largest singular values of the full channel matrix, and therefore benefits from a better SNR compared with HS-B without PSS-opt. For the PSS-sopt the linearly transformed channel matrix is

$$
\boldsymbol{H}_{\text {PSS-sopt }}=\frac{\left|\boldsymbol{\Phi}_{\boldsymbol{U}_{\boldsymbol{H}}}^{\dagger} \boldsymbol{H} \boldsymbol{\Phi}_{\boldsymbol{V}_{\boldsymbol{H}}}\right|^{2}}{N_{\mathrm{T}}}
$$

from which the $\left(L_{\mathrm{R}}, L_{\mathrm{T}}\right)$ largest singular values are used for capacity evaluation (4). $\Phi_{\boldsymbol{U}_{H}}$ and $\Phi_{V_{\boldsymbol{H}}}$ are simple suboptimal solutions for the optimal matrices $\boldsymbol{U}_{\boldsymbol{H}, 1}$ and $\boldsymbol{V}_{\boldsymbol{H}, 1}$, including only the phase information of the entries in $\boldsymbol{U}_{\boldsymbol{H}, 1}$ and $\boldsymbol{V}_{\boldsymbol{H}, 1}$.

\subsection{FFT preprocessing and selection (FFTS)}

The FFT preprocessing can be viewed as a special case of the PSS, where the preprocessing matrix is a Butler matrix (FFT-matrix). Thus, the signal is spatially Fourier-transformed before downconversion and selection, see for example [10]. Note that the values of the phase shifters in the Butler matrix are time-invariant and cannot be adjusted according to the channel state. The FFT preprocessing scheme has been shown to work well for uniform linear arrays and highly correlated channels.

\section{Measurement setup}

For the performance analysis we use measurements of MIMO channels in an access point-to-PC/laptop (AP-PC) scenario at $2.6 \mathrm{GHz}$ and handheld-to-handheld ( $\mathrm{HH}-\mathrm{HH})$ scenario at $5.2 \mathrm{GHz}$ (In [24] the AP was referred to as 'fixed device'.). The measurements were performed with the RUSK LUND channel sounder, and the total available bandwidth of $200 \mathrm{MHz}$ was divided into 321 frequency points (sub-channels). The length of the test signal, as well as the guard period between successive test signals, was set to $1.6 \mu \mathrm{s}$ corresponding to a resolvable excess delay of $480 \mathrm{~m}$, which was enough to avoid significant inter-symbol interference between the transmitted test signals. Because of the short distances measured, and the high dynamic range of the measurement equipment, all measurement results showed a very high dynamic range and an SNR above $20 \mathrm{~dB}$.

The access point was a dual-polarised $(4 \times 8 \times 2)($ rows $\times$ columns $\times$ polarisations) patch array (Fig. $2 a$ ). During the measurements, only the middle two rows $(2 \times 8 \times 2)$ were used, and all unused elements were terminated with 

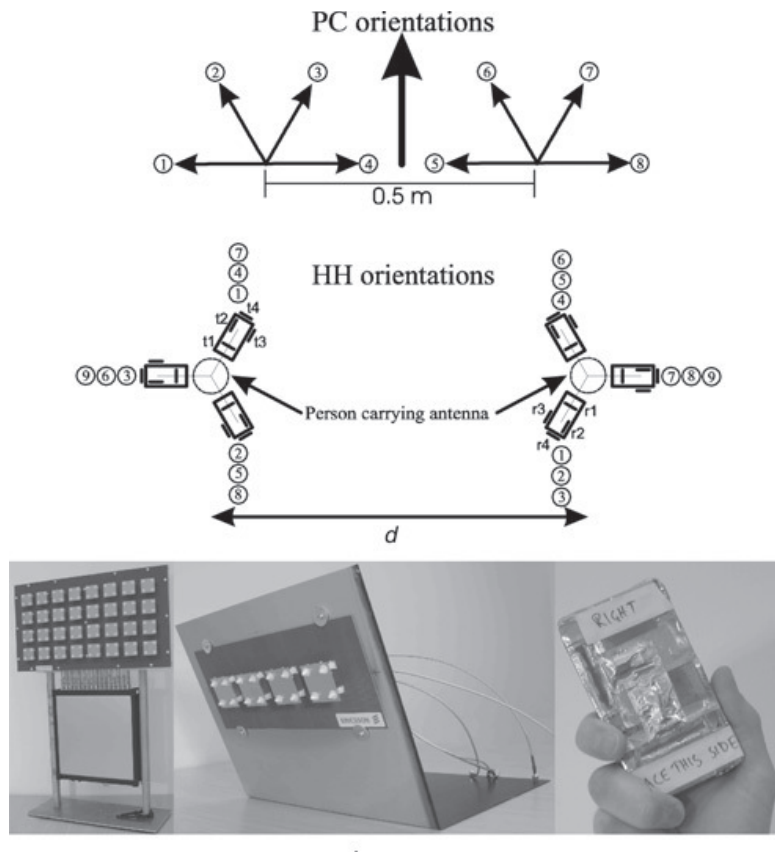

a

c

Fig. 2 Laptop (PC) and handheld (HH) orientations

Thick arrow is a reference also used in site map (see Fig. 1) for the PC Pictures of: $(a) \mathrm{AP},(b) \mathrm{PC}$ and $(c)$ one of the two identical HH devices

$50 \Omega$-terminations. The AP array was tripod-mounted at a height close to the ceiling in order to increase the resemblance with a real AP. The PC had an $(1 \times 4 \times 2)$ array, consisting of the same sort of elements as the AP, mounted on the back of the 'screen', with the broadside direction aiming in the opposite direction of the 'keyboard', (Fig. 2b). Since the 'screen' is slightly tilted backwards $\left[110^{\circ}\right.$ with respect to the 'keyboard', (Fig. 2)] in order to represent a typical laptop setup, so is the antenna array.

For the HH-HH measurements, two identical hand-held devices were used, each made of a metal box with four slot antennas. Two slot antennas are at the front of the box, perpendicular to each other, one is mounted at the top side and one is at the right side of the box (Fig. 2c).

The measurements were performed in an office environment in the E-building at LTH, Lund University, Sweden. The building is made of reinforced concrete with gypsum wallboards separating the different offices. Two different TX-RX positions were used for AP-PC measurements and two TX-RX positions for HH-HH (one for LOS and one for NLOS). In this paper, we define LOS as any measurement where there is a direct optical path between the TX and the RX devices or the persons holding the devices. Hence, LOS also includes cases where some or all antenna elements are obstructed by the device or by the person carrying the device.

Measurements for a number of different orientations of the $\mathrm{PC}$ and the $\mathrm{HH}$ were taken for each $\mathrm{TX}-\mathrm{RX}$ position. The orientations are shown in Fig. 2. More details about the measurement setup can be found in [24, 29]. For each orientation, the channel gains are normalised; that is, we assume a certain average receive power, where the averaging is done over the frequency range and the small-scale movements of the devices.

\section{Algorithm comparison}

In this section the average normalised SNR gain and capacity for seven different antenna selection schemes (see section 3) are presented for our measured channels, as well as i.i.d. model channels. Both the AP-PC (verticalpolarised) and $\mathrm{HH}-\mathrm{HH}$ scenario are considered. The same antenna selection algorithm was used at both the ends for LOS, NLOS and i.i.d. scenarios. Figs. 3 and 4 analyse AP-PC setups with $8 \times 4$ vertically polarised elements on both ends in line configuration, and $\mathrm{HH}-\mathrm{HH} 4 \times 4$ arrangements. When eight antenna elements are available, four are selected; when four are available, two are selected.

Let us first consider the results for diversity systems. For the FC scheme, on an average, the AP-PC scenario offers an SNR gain of $2.5 \mathrm{~dB}$ compared with the $\mathrm{HH}-\mathrm{HH}$ case. In a pure LOS scenario, we would expect a $3 \mathrm{~dB}$ difference, since the AP scenario has twice the number of antennas [26]. However, the SNR from MRC/MRT is distributed according to the largest singular value distribution of the MIMO channel, hence only for MIMO channels of rank one (e.g. pure LOS and keyhole channels) the full beamforming gain $N_{\mathrm{R}} \cdot N_{\mathrm{T}}$ is possible; therefore the gain from the higher number of RX antennas in the AP-PC case is reduced.

For antenna selection with PSS preprocessing, we find that the PSS-opt algorithm performs as well as the FC scheme; this is in line with the predictions of [11]. However, the performance gap between PSS-opt and PSS-sopt varies greatly. In the AP-PC scenario, the performance gap is negligible, while in the HH-HH case, it can be more than $1 \mathrm{~dB}$. This is because the amplitude variation of the AP-PC is smaller when compared with $\mathrm{HH}-\mathrm{HH}$. Therefore the sub-optimal PSS algorithm (equivalent to EGC), where all elements are weighted with the same power, will give more similar results to PSS-opt (MRC) in the AP-PC than in the HH-HH scenario.

Next, we compare the performance of the 'standard' hybrid selection (HS-B) to the performance of the FFT-based selection. As predicted by theory, there is no difference in performance in i.i.d. channels. Similar results hold for the NLOS case of the AP-PC scenario. In the LOS case of the AP-PC scenario, we find an

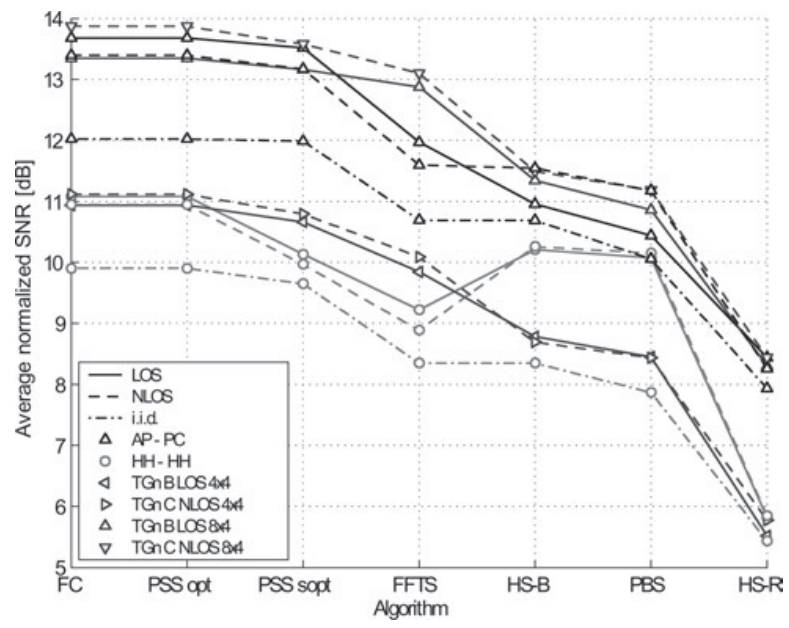

Fig. 3 Average normalised SNR for the different antenna selection schemes are presented

Both AP-PC $(8: 4 \times 4: 2$ vertical polarised elements on both ends, line configuration) and $\mathrm{HH}-\mathrm{HH}(4: 2 \times 4: 2$ single polarised elements $)$ results are presented for LOS (rotation 2, 3, 6, 7 for AP-PC V-polarised and 1, 2, 4, 5 for $\mathrm{HH}-\mathrm{HH}$, due to high $K$-factor) and NLOS (rotation 3, 4, 7, 8 for AP-PC and 9 for HH-HH due to low $K$-factor)

Same antenna selection algorithm was used at both ends

For comparison the TGn B for LOS and TGn C for NLOS together with i.i.d. channel results are presented 


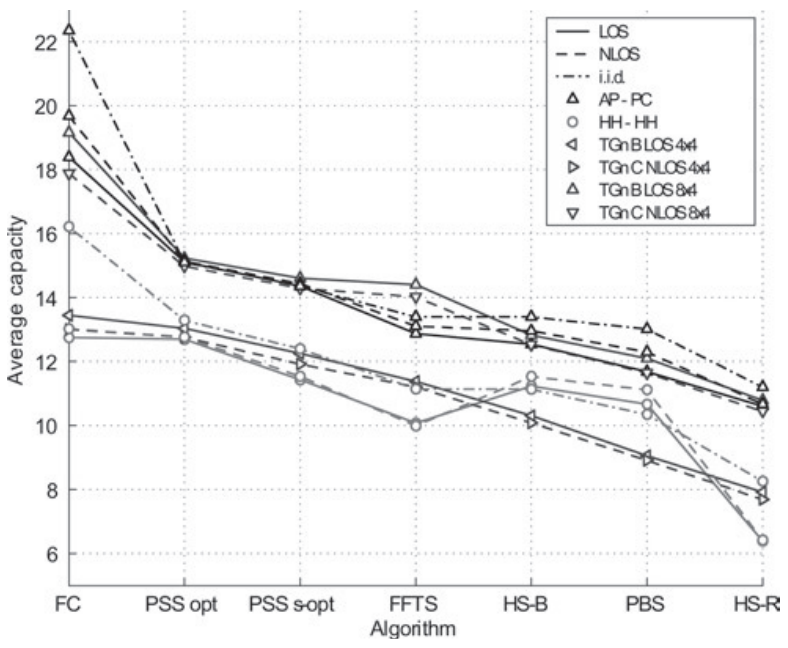

Fig. 4 Average capacity for the different antenna selection schemes are presented

Both AP-PC $(8: 4 \times 4 \times 2$ vertical polarised elements on both ends, line configuration) and $\mathrm{HH}-\mathrm{HH}(4: 2 \times 4: 2$ single polarised elements $)$ results are presented for LOS (rotation 2, 3, 6, 7 for AP-PC V-polarised and 1, 2, 4, 5 for $\mathrm{HH}-\mathrm{HH}$, due to high $K$-factor) and NLOS (rotation 3, 4, 7, 8 for AP-PC and 9 for $\mathrm{HH}-\mathrm{HH}$ due to low $K$-factor)

Same antenna selection algorithm was used at both ends

For comparison the TGn B for LOS and TGn C for NLOS together with i.i.d. channel results are presented

improvement by using an FFT of about $1 \mathrm{~dB}$. This improvement is not as strong as theoretically predicted for LOS channels under ideal circumstances; the deviation can be explained by the fact that the LOS channels do not have a very high Rice $K$-factor [29], and the direction of the LOS component does not coincide with the maximum of the formed beams.

For the HH-HH scenario, we find that the FFTS considerably decreases the SNR, both for the LOS and the NLOS case. The reason for this effect are 2-fold: (i) the antennas on the handsets are not arranged in a linear form; therefore the beampatterns formed by the FFT point into arbitrary directions; (ii) the average power received on the different antenna elements is unequal. Therefore the spatial FFT preprocessing smears out the already-concentrated power, resulting in performance loss compared with no preprocessing (e.g. HS-B).

Note also that AP-PC without preprocessing, for example HS-B and PBS, performes better in an NLOS compared with an LOS, because of the larger variations in received power of the elements in NLOS scenario.

The large average power differences between the elements in the HH-HH scenario also result in a severe penalty when the good element(s) are not in the selected subset (compare HS-B and HS-R). Hence, the selection algorithm is more important for configurations with large average power differences between the elements. Even when picking the worst possible element subset in the AP-PC scenario the gain is at least $2 \mathrm{~dB}$ compared with a SISO system, but for the HH-HH, HS-W performes worse than SISO systems, again due to the average power variations of the elements (results not shown here due to space constraints).

Finally, we notice that power-based selection works well for almost all scenarios. Especially for the HH-HH case, power-based selection performs very similar to HS-B. Again, this can be explained by the fact that the different antenna elements receive different average power.
In Fig. 4 the average capacity for seven different antenna selection schemes are presented. Both the AP-PC and $\mathrm{HH}-\mathrm{HH}$ scenario are considered. We first observe that the capacity of the FC scheme is considerably higher than with any of the antenna selection/preprocessing schemes; this is due to the larger number of spatial streams that can be transmitted.

For the AP-PC scenario, we find that (imposing equal receive SNR for all cases) the smaller the Rice $K$-factor, and the larger the angular spread, the higher the capacity - a result that is well known. However, for $\mathrm{HH}-\mathrm{HH}$, the non-uniform average element power results in better performance for the LOS and NLOS compared with the i.i.d. for HS-B and PBS.

Optimum preprocessing before HS-B (PSS-opt) results in $13 \%$ and $18 \%$ capacity increase compared with HS-B only for the AP-PC and $\mathrm{HH}-\mathrm{HH}$ scenario, respectively. For HH-HH, i.i.d. PSS-sopt performes better than HS-B, which is not the case for LOS and NLOS.

Most of the other trends for the average capacity parallel the trends of the SNR discussed above, and can be explained in an analogous manner.

A general behaviour of the capacity evaluation of the selection schemes is that for low SNRs, where only a few eigenmodes are contributing to the capacity, the PSS-opt selection scheme performs better than the FC scheme. The capacity equation assumes no channel state information (CSI) at the TX (no waterfilling), but antenna selection uses a certain amount of CSI.

\section{Bulk- and per-tone selection}

In this section, we investigate the performance of antenna selection used in a frequency selective MIMO-OFDM system. Such a system is a more realistic scenario for broadband wireless local area network (WLAN) settings. In this scenario, the system model becomes frequency selective.

We investigate two types of antenna selection: (i) bulk selection, where the selected antenna subset is used for all OFDM sub-channels and (ii) per-tone selection, where a different element subset can be used for each tone. Naturally, the second solution requires a much higher complexity; the signals from all antenna elements have to be converted to/from baseband, and the selection is implemented in baseband.

For the AP-PC setup $(8: 4 \times 4: 2)$ there are $\left(\begin{array}{l}8 \\ 4\end{array}\right) \cdot\left(\begin{array}{l}4 \\ 2\end{array}\right)=420$ available subsets and for $\mathrm{HH}-\mathrm{HH}$ $(4: 2 \times 4: 2)$ there are 36 available sub-sets. The bulk selection is made from either an extensive search of all possible subsets (bulk opt) or by averaging the power over all frequency sub-channels and then apply PBS (bulk PBS). We also evaluate the performance of bulk PBS with an FFT preprocessing scheme (bulk FFT PBS).

In case of per-tone selection, the sequence length of the number of adjacent sub-channels selecting the same subset depends on the channel characteristics, and affects the performance of bulk selection schemes discussed previously. In order to study the HS-B subset variation with the orthogonal frequency division multiplexing (OFDM) sub-channels, we show the cumulative distribution function (CDF) of sequence lengths (from HS-B) in Fig. 5. In agreement with intuition, the LOS scenarios (large coherence bandwidth) have more long sequences, that is sequences with many sub-channels selecting the same subset, compared to NLOS. For example in the AP-PC LOS scenario, $50 \%$ of the sequences have length 10 or more. Of course, this value depends on the tone spacing (0.62 MHz in our case), and the power delay profile of the 

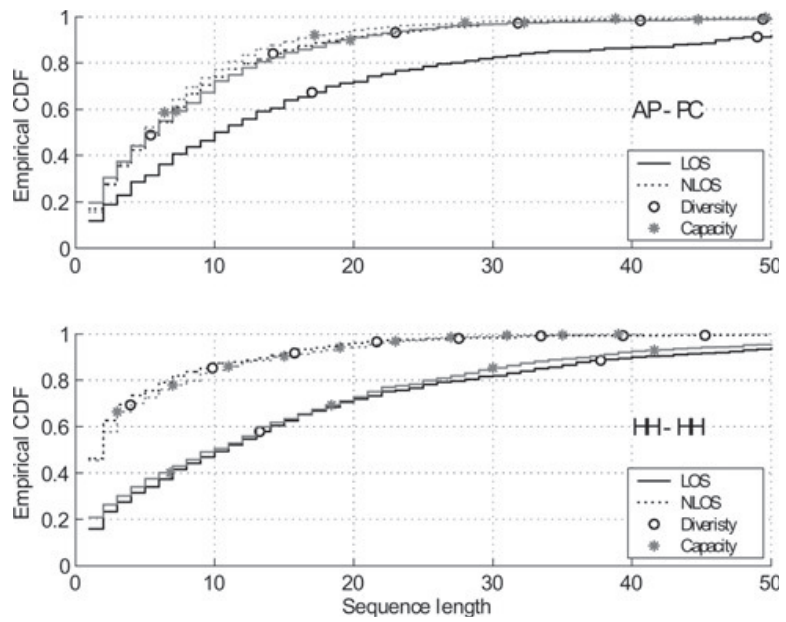

Fig. 5 CDFs of the sequence length

Where sequence length is the number of consecutive sub-channels (a total of 321 corresponding to $200 \mathrm{MHz}$ ) that are using the same subset (HS-B) for AP-PC (dual-polarised elements at both ends) and HH-HH Both LOS and NLOS scenarios are considered

channel. For the AP-PC LOS scenario, there are measurement points with a sequence length of 321, that is, where the same antenna subset is optimum for all 321 sub-channels. Note that our AP-PC LOS scenario has rather small $K$-factor; a purer LOS will have even less variations of subsets over the subchannels. In the AP-PC LOS scenario, the subsets chosen for maximising capacity has fewer long sequences than the subset selected for maximising diversity. This difference is not that evident in the $\mathrm{HH}-\mathrm{HH}$ scenario, probably due to the larger average power variations of the $\mathrm{HH}$ elements, and because of the inherently low correlation of the $\mathrm{HH}$ antenna elements (pointing in different directions).

Fig. 6 shows the CDFs of the normalised SNR, comparing bulk selection and per-tone selection. Since the SNR presented in these figures is averaged over the subchannels, the results include frequency diversity gain. Both LOS and NLOS for
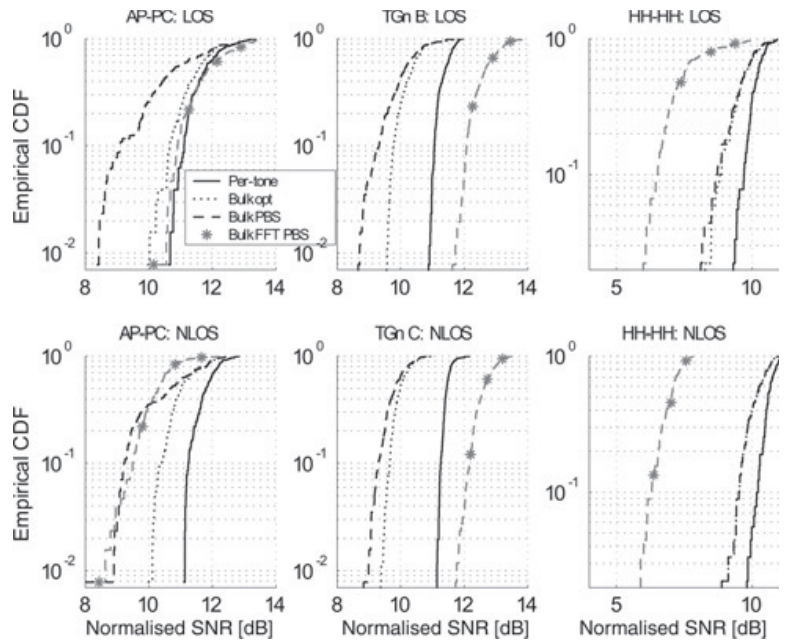

Fig. 6 CDF of normalised SNR averaged over sub-channels (i.e. frequency diversity included)

AP-PC, TGn $(b, c)$ and HH-HH for both LOS and NLOS scenarios (configurations for AP-PC line: H, V, Alt, HV, DP) 8:4 × 4:2

Antenna subset selection is either found per sub-channel (per-tone selection) or the same subset is used for all sub-channels (bulk selection PBS or bulk selection optimal)

Frequency bandwidth is $200 \mathrm{MHz}$

Effect of FFT preprocessing is also presented
AP-PC, HH-HH and two theoretical channel models (model $\mathrm{B}$ and $\mathrm{C}$ of the IEEE 802.11.TGn models [23]) are evaluated. The TGn models are commonly used for modelling AP-PC scenarios, where the shorter distance LOS scenario is modelled by TGn B (LOS) and an NLOS scenario with a somewhat larger distance is modelled by TGn C (NLOS).

The results show that in the AP-PC scenario, bulk opt selection has a smaller performance loss (compared to per-tone selection) for LOS than NLOS, because of the smaller variations in the used subsets (Fig. 5). However, power-based bulk selection has about similar performance loss in both the LOS and the NLOS scenario. FFT preprocessing results in a significant performance gain compared with bulk PBS for the AP-PC LOS. This can be explained by the fact that the FFT forms beams, one of which points (approximately) into the direction of the LOS. Consequently, there are fewer variations in the optimal subset between different frequency sub-channels - the beam pointing towards the LOS will almost always be chosen.

When analysing the FFT scheme operating in TGn channel models, we find extremely high gains. The TGn models uses 9 and 14 MPC (B and $\mathrm{C}$, respectively) that are divided into two clusters and the FFT preprocessing is able to put beams in those directions; the limited spread of the clusters in the TGn models, results in a high gain for the FFT preprocessing. Consequently, the array gain for those models differs significantly from the measurement results, where the spread of the MPC are larger.

Without preprocessing, the TGn models generate results similar to the AP-PC measurements. It is therefore important to note that the relative performances of the selection schemes as estimated from the TGn models can differ significantly from the results of the measurements.

For HH-HH, bulk PBS and bulk opt selection perform similar. This is a result of the non-uniform average power distribution of the $\mathrm{HH}$ elements: it is usually best to select the antennas that have the largest power. The average power variation decreases the performance of the bulk FFT PBS scheme since, the spatial FFT preprocessing results in a power smearing.

Also note that the frequency selectivity results in a frequency diversity gain, hence the low variance of the normalised SNRs. For a smaller bandwidth or higher coherence bandwidth the difference between per-tone and bulk selection will decrease.

The capacity evaluations shows quantitatively similar results to the SNR case and the figures are left out due to space limitations.

\section{Shadowing effects and antenna configuration}

In this section, we investigate a number of different configurations for the antenna elements on the AP, to find the most suitable one for antenna selection algorithms. The AP consists of two stacked ULAs (Section 4) with dualpolarised patch elements. The configurations evaluated at the AP are (Fig. 7): (i) 'Line', which is an ULA configuration taken from one of the AP's two ULAs; (ii) 'Saw', where the elements alternate from the AP's two ULAs; and (iii) 'Rectangular' where the elements are taken in pairs from the AP's two ULAs. For each configuration, four different polarisation schemes are evaluated (Fig. 7): (i) all elements in the configuration are horizontally $(\mathrm{H})$ polarised; (ii) all elements in the configuration are vertically (V) polarised; (iii) the polarisation alternated between $\mathrm{H}$ and V (Alt HV); and (iv) all elements in the configuration are dual-polarised (DP). The PC is a linear half wavelength dual-polarised array for all AP configurations. 


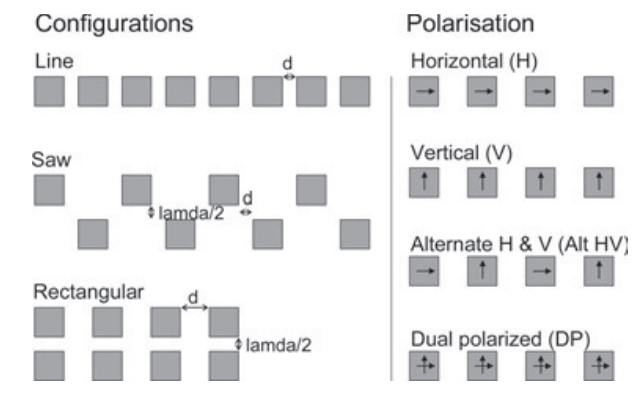

Fig. 7 AP array configurations and polarisations $\mathrm{PC}$ is dual-polarised for all AP configurations and polarisations

To capture the effect of large-scale fading (created by different PC orientation) and small-scale fading, the measured channel is normalised in two ways: (i) Large-scale power control; the received array power between each measurement position (including eight orientations, all antenna elements and all polarisations) is constant, so that the received array power shows variations due to different configurations and polarisations, and (ii) Small-scale power control; the received array power between each rotation, polarisation and configuration is constant, that is, variations due to PC orientation and polarisation are normalised out.

\subsection{Diversity results}

In Fig. 8, the effects from large- and small-scale power control, array configuration and polarisation on the normalised SNR are presented. Results are only shown for the HS-B selection scheme.

For the LOS scenario, the polarisation effect is more pronounced for the large-scale fading. Vertical polarisation is the best choice for all configurations (except for the 'Rectangle'), whereas the horizontal polarisation performs worst for all configurations. For small-scale power control, there is almost no variation with polarisation, hence there are
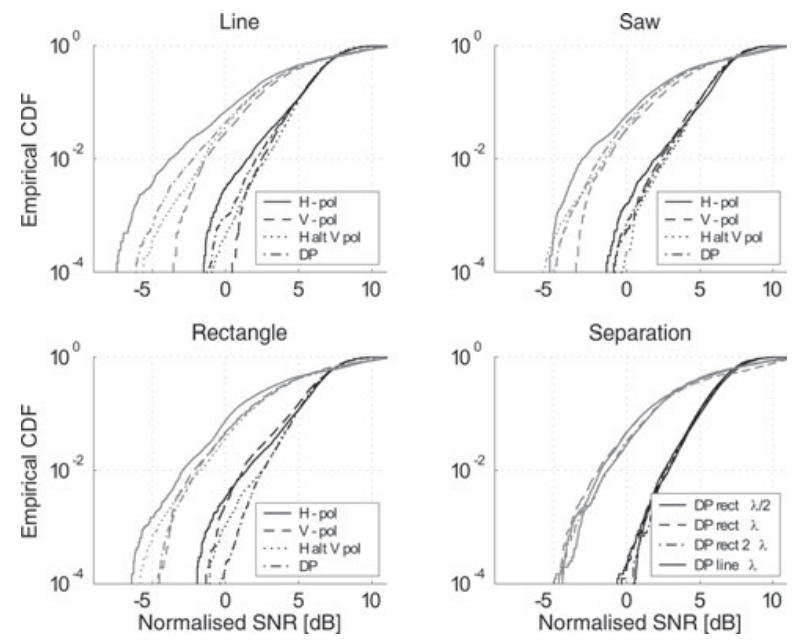

Fig. 8 Configuration comparison for the AP-PC scenario HS-B at PC only

LOS. $4: 2 \times 2: 2$

Including shadow fading results (grey) and results where the rotation effect of the PC is normalised out (black)

Lower right subplot shows the element separation effects on normalised SNR

$\mathrm{PC}$ is DP for all configurations differences in received power between the polarisations, resulting in a difference in the normalised SNR for the large-scale power control case but not for the small-scale power control case. The different array configurations do not seem to matter in terms of diversity gain, but the Saw configuration shows the least sensitivity to the polarisation.

For NLOS (not presented here due to space limitations), the polarisation effect is less pronounced compared with LOS. This result follows intuition - the interaction of the multipath components with surrounding objects, which are dominant in NLOS, tend to depolarise the radiation. The shadowing variations due to rotation of the PC is larger for the NLOS case, probably due to specific properties of the chosen measurement locations [for rotation 4 and 8 , the $\mathrm{PC}$ is directed towards the AP, and for rotation 1 and 5 the $\mathrm{PC}$ is directed away from the AP (Fig. 1)] compared with LOS where the PC goes from endfire towards the AP to endfire away from the AP.

We also observe that increasing the horizontal spacing of the antenna elements does not improve the SNR in either LOS and NLOS. This result is different from the conclusions we would get from the TGn channel models.

Next, we analyse the impact of large- and small-scale power control for the HH-HH scenario (Fig. 9) in both LOS and NLOS. The HH device has elements pointing in different directions. Therefore antenna selection can help reduce the shadowing due to rotation. This conjecture is supported by the results shown in Fig. 9: the difference between the large-scale fading and small-scale fading is small compared with the AP-PC scenario. The large variation of average power of the $\mathrm{HH}$ elements make the selection important: the diversity gain (compared with no selection) is up to $40 \mathrm{~dB}$ at an outage probability of $0.01 \%$. The 'saddle point' for the $1: 1 \times 1: 1$ curve is due when the used element is one with a small average power. Note also that the slope of the SNR distribution is steeper in the HH-HH scenario than in the AP-PC scenario. This again can be explained by the better ability of the $\mathrm{HH}$ antenna arrangement to combat shadowing.

\subsection{Capacity results}

In Fig. 10, the effects from large- and small-scale power control, array configuration and polarisation on capacity (when HS-B is used) are presented.
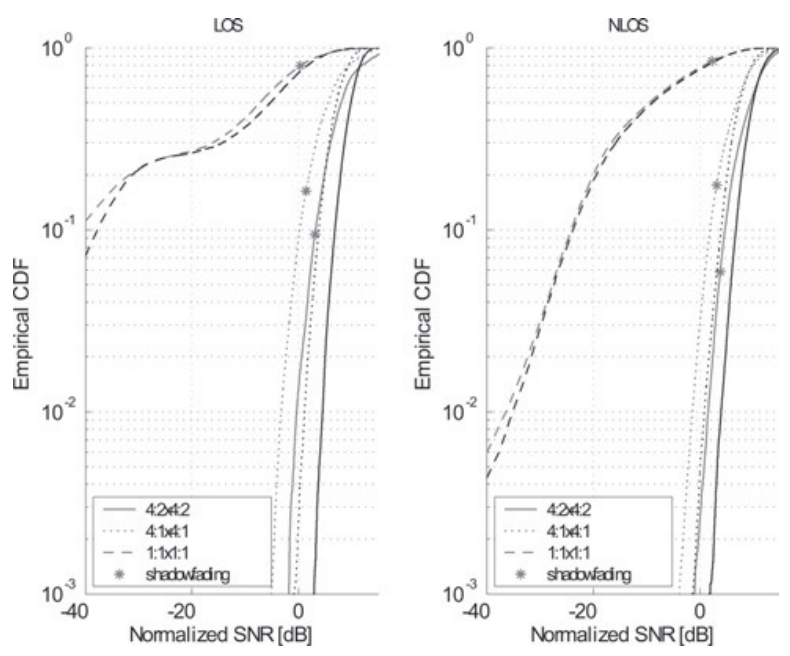

Fig. 9 HH-HH including rotation shadow fading and no shadow fading for $4: 2 \times 4: 2,4: 1 \times 4: 1$ and $1: 1 \times 1: 1$ 

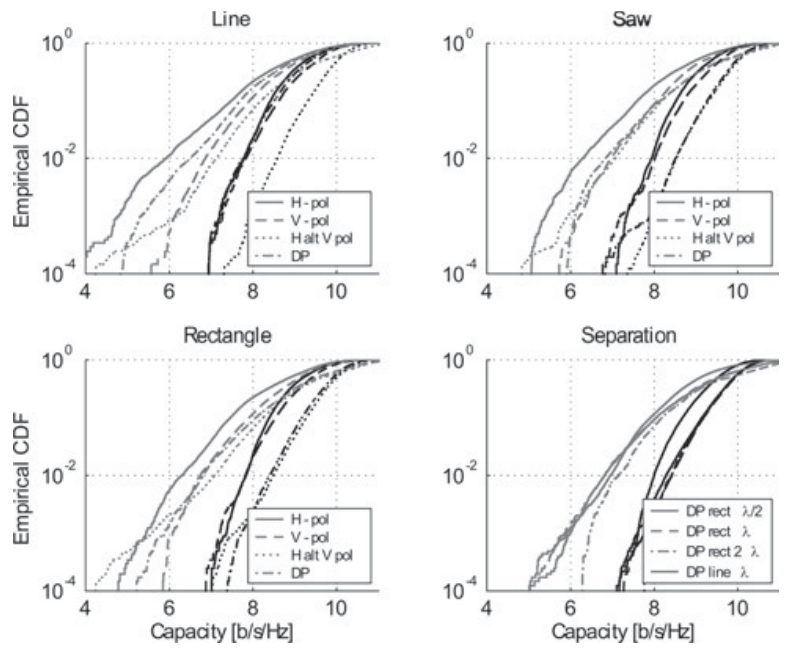

Fig. 10 Configuration comparison for the AP-PC scenario $H S-B$ at PC only

LOS. $4: 2 \times 2: 2$. Including shadow fading results (grey) and results where the rotation effect of the PC is normalised out (black)

Lower right subplot shows the element separation effects on capacity $\mathrm{PC}$ is DP for all configurations

Compared with diversity, the polarisation effect is more pronounced when studying the capacity. For the small-scale power control case (when the power difference between polarisations is normalised out), the polarisation effect is still noticeable, hence the propagation characteristics for the different polarisations does affect the capacity more than the normalised SNR.

For both the LOS and the NLOS (not presented) scenarios 'Alt HV' seems to be the best polarisation solution, since the different polarisations are almost independent and the distance between neighbouring elements with the same polarisation is the largest. This in turn results in a lower correlation; 'Alt HV' could be viewed as the 'DP' configuration but with a larger elements separation, resulting in a slightly better performance compared with DP. As for the normalised SNR, especially for LOS, the horizontal polarisation has the worst performance in capacity sense.

For the NLOS (not presented here due to space limitations), the less polarisation influence, due to the depolarisation effects of the environment discussed above.

The different array setups do not seem to matter in terms of capacity (similar result as for the diversity evaluation). The impact of element spacing is also almost negligible, a small favour of the largest separation in the case with rotation shadowing is discerned. It is noteworthy that there is almost no difference in terms of capacity between the LOS and NLOS scenarios, probably due to the large variations of the $K$-factor in the LOS scenario. We also note that the rectangular configuration has a similar capacity as the in-line configuration. This result would not be obtained in the TGn channel models, because they do not include elevation spread, and thus would lead to strong correlation between vertically stacked antenna elements.

\section{Discussions and conclusions}

We have performed an experimental evaluation of the closed-loop diversity gain and open-loop capacity that can be achieved by MIMO systems with antenna selection. Our evaluations were performed in WLAN and WPAN scenarios for AP-PC and $\mathrm{HH}-\mathrm{HH}$ communication. Seven different antenna selection schemes and preprocessing methods were evaluated based on measurement data. Our main results can be summarised as follows:

- The FFT preprocessing decreases the diversity and capacity performance for array configurations with unequal average power at the different antenna elements.

- The polarisation effect on diversity gain is negligible, but evident for capacity evaluations.

- The three AP array configurations (Line, Rectangle, Saw) did not have any significant effect on diversity or capacity results.

- The TGn channel model is not able to model all relevant effects observed in the experiments. This is especially true when analysing the FFT preprocessing scheme.

- For the wideband scenario, the FFT preprocessing scheme helps to reduce the difference between per-tone selection and bulk selection.

- The PSS-sopt performs well in situations where the average elements power is similar, but performs worse than HS-B when the average element power is different.

- The HH construction with elements pointing into different directions, used together with HS-B, combats the large-scale fading very well.

Those derivations can be seen as guidelines for the design of antenna arrays for antenna selection in practical scenarios.

\section{Acknowledgments}

The authors would like to thank Dr. Neelesh B. Mehta for helpful discussions and comments and NEWCOM and Bristol University for making it possible for us perform measurements with their $\mathrm{HH}$ devices. This work was partly funded by an INGVAR grant of the Swedish Foundation for Strategic Research, a grant from Vetenskapsrådet, and the SSF center of Excellence for High-speed Wireless Communications.

\section{References}

1 Winters, J.H.: 'On the capacity of radio communications systems with diversity in Rayleigh fading environments', IEEE J. Select. Areas Commun., 1987, 5, pp. 871-878

2 Foschini, G.J., and Gans, M.J.: 'On limits of wireless communications in fading environments when using multiple antennas', Wireless Personal Commun., 1998, 6, (3), pp. 311-335

3 Win, M.Z., and Winters, J.H.: 'Analysis of hybrid selection/ maximal-ratio combining in Rayleigh fading', IEEE Trans. Commun., 1999, 47, pp. 1773-1776

4 Molisch, A.F., Win, M.Z., and Winters, J.H.: 'Reduced-complexity transmit/receive-diversity systems', IEEE Trans. Signal Process., 2003, 51, pp. 2729-2738

5 Molisch, A.F., Win, M.Z., Choi, Y.S., and Winters, J.H.: 'Capacity of MIMO systems with antenna selection', IEEE Transactions on Wireless Communications, 2005, 4, (4), pp. 1759-1772

6 Heath, R.W.J., Sandhu, S., and Paulraj, A.J.: 'Antenna selection for spatial multiplexing systems with linear receivers', Commun. Lett., 2001, 5, pp. $142-144$

7 Gore, D.A., and Paulraj, A.J.: 'MIMO antenna subset selection with space-time coding', IEEE Trans. Signal Process., 2002, 50, pp. $2580-2588$

8 Gorokhov, A.: 'Antenna selection algorithms for MEA transmission systems'. Proc. IEEE Int, Conf., on Acoustics, Speech, and Signal Processing (ICASSP), 13-17 May 2002, vol. 3, pp. 2857-2860

9 Gharavi-Alkhansari, M., and Gershman, A.B.: 'Fast antenna subset selection in wireless MIMO systems'. Proc. IEEE Int. Conf., on Acoustics, Speech, and Signal Processing (ICASSP), 2003, vol. 5, pp. $57-60$

10 Molisch, A.F., and Zhang, X.: 'FFT-based hybrid antenna selection schemes for spatially correlated mimo channels', IEEE Commun. Lett., 2004, 8, pp. 36-38 
11 Zhang, X., Molisch, A.F., and Kung, S.-Y.: 'Variable-phaseshift-based RF-baseband codesign for MIMO antenna selection', IEEE Trans. Signal Process., 2005, 53, pp. 4091-4103

12 Choi, Y.-S., and Alamouti, S.: 'Performance analysis and comparisons of antenna and beam selection diversity'. Proc. IEEE VTC $2004-$ Fall, IEEE, 2004, vol. 1, pp. 165-170

13 Coffey, S., Kasher, A., and Stephens, A.: 'Joint proposal: High throughput extension to the 802.11 standard: PHY', IEEE 802.1105/1102r4, January 2006

14 Zhang, H., Molisch, A.F., and Zhang, J.: 'Applying antenna selection in WLANs for achieving broadband multimedia communications', IEEE Trans. Broadcast., 2006, 52, pp. 475-482

15 Molisch, A.F., and Win, M.Z.: 'MIMO systems with antenna selection', IEEE Microwave Mag., 2004, 5, pp. 46-56

16 Sanayei, S., and Nosratinia, A.: 'Antenna selection in MIMO systems', IEEE Commun. Mag., 2004, 42, pp. 68-73

17 Mehta, N.B., and Molisch, A.F.: 'Antenna selection' in Tsoulous, G. (Ed.): 'MIMO System Technology For Wireless Communications' Taylor \& Francis, CRC press, 2006, pp. 147-174

18 Casas E., Chia T., da Silva M., Yin H., and Choi, Y.-S. 'Beam diversity for indoor WLAN systems'. IEEE VTC, 2003, 5, pp. $3141-3144$

19 Jiang, J.-S., and Ingram, M. A.: 'Comparison of beam selection and antenna selection techniques in indoor MIMO systems at $5.8 \mathrm{GHz}$ '. Proc. Radio and Wireless Conf. (RAWCON), 10-13 August 2003, vol. 1, pp. 179-182

20 Grau, A., Romeu, J., Blanch, S., Jofre, L., and De Flaviis, F. 'Optimization of linear multielement antennas for selection combining by means of a butler matrix in different MIMO environments', IEEE Trans. Antennas Propag., 2006, 54, pp. 3251-3264

21 Onizawa, T., Ohta, A., Asai, Y., and Aikawa, S.: 'Experimental evaluation of transmit antenna selection implemented in FPGA for eigenbeam MIMO-OFDM'. Proc. IEEE PIMRC, 2006, pp. 1-5

22 Almers, P., Santos, T., Tufvesson, F., Molisch, A.F., Karedal, J., and Johansson, A.: 'Measured diversity gains from mimo antenna selection'. Proc. VTC 2006 Fall, IEEE, September 2006, pp. 1-6

23 Erceg, V., Schumacher, L., Kyritsi, P., Molisch, A.F., Baum, D.S., et al.: 'TGn channel models'. IEEE 802.11-03/940r4, May 2004

24 Johansson, A., Karedal, J., Tufvesson, F., and Molisch, A.F.: 'MIMO channels measurements for personal area networks'. Proc. Vehicular Technology Conf. 2005 spring, IEEE, May-June 2005, vol. 1, pp. $171-176$

25 Molisch, A.F.: 'Wireless Communications' (IEEE Press, Wiley, 2005, 1st edn.)

26 Andersen, J.B.: 'Antenna arrays in mobile communications: gain, diversity, and channel capacity', IEEE Antennas Propag. Mag., 2000, 42, (2), pp. 12-16

27 Lo, T.K.Y.: 'Maximum ratio transmission', IEEE Trans. Commun., 1999, 47, pp. $1458-1461$

28 Vaughan, R., and Andersen, J.B.: . Channels, propagation and antennas for mobile communications, 2003, (IEE Press, London, UK, 1st edn.)

29 Karedal, J., Johansson, A.J., Tufvesson, F., and Molisch, A.F.: 'Characterization of MIMO channels for handheld devices in personal area networks at $5 \mathrm{GHz}$. Proc. European Signal Processing Conf. 2006, Florence, Italy, September 2006, Invited paper 